\title{
A unique case of dysferlinopathy with a large-segment duplication mutation who experienced rapid deterioration after small-dosage corticosteroid treatment
}

$\mathrm{Li} \mathrm{Di}^{1}$, Yuqing Guan ${ }^{2}$, Xin-Ming Shen ${ }^{3}$, Yuwei $\mathrm{Da}^{1}$

Keywords: dysferlinopathy, duplication mutation, corticosteroid, deterioration

Dear Editor,

Dysferlinopathy is a type of myopathy with predominant deficiency of the dysferlin protein caused by mutations in the DYSF gene. The patients have defective skeletal muscle membrane repair. Dysferlinopathy is a spectrum of autosomal recessive neuromuscular disorders. To date, more than 450 different mutations have been reported in the DYSF gene, most being missense or nonsense mutations (http://www.dmd.nl). Because of the relatively acute onset and the remarkable inflammatory changes observable in muscle biopsies, dysferlinopathy has a big chance of being misdiagnosed as an inflammatory myopathy, and 
the patient consequently being prescribed steroid treatment[1]. Here we present a case of dysferlinopathy with a large-segment duplication mutation, in which the patient was given a small-dosage steroid therapy and experienced rapid deterioration.

\section{Case Report}

A 20-year-old female had slowly progressing lower limb weakness for 5 years. She noticed that she ran slower than her peers and she could not walk on her toes at age 15. At the age of 18 , she went to the local hospital because medical tests had shown abnormal levels of transaminase. A neurological examination revealed normal cranial nerve and upper limbs function. The muscle strength was 5 of 5 in the proximal lower limbs, while it was 3 of 5 in the distal lower limbs with atrophy of muscles in both distal lower limbs. Her serum CK was 917U/L (reference range:22-198 U/L), aspartate aminotransferase was 164U/L (reference range: 10-40 IU/L) and alanine aminotransferase was 189IU/L (reference range: 9-60 IU/L). Autoimmune antibodies and viral antibodies were normal. Electromyography (EMG) showed myogenic patterns in the deltoids, tibialis anterior and rectus femoris. A muscle biopsy was taken from her right quadriceps, which showed myogenic damage with mild proliferation in connective tissue and variations in fiber size, concomitant with obvious degeneration, necrosis and regeneration. Inflammatory infiltrate was absent. She was treated with oral methylprednisolone $16 \mathrm{mg}$ per day. Five days after the initiation of steroid treatment, the patient complained about worsening weakness. Within two weeks of the treatment she had difficulty in walking upstairs and could not stand up from a squatting position. Remarkable elevation of the serum CK level to $23722 \mathrm{U} / \mathrm{L}$ was discovered, corresponding to the clinical deterioration. The patient denied strenuous exercise or any symptoms of a viral illness or intake of any other medicine or health products during that period. She stopped taking methylprednisolone and refused to do any test. Her weakness partly improved in the next one year. She did not report any history of other diseases and her family history was unremarkable. She had normal milestones in development. On examination, she was cognitively normal, the cranial nerve and upper limb function were normal, whereas the proximal lower limbs were grade 4 and distal lower limbs were grade 3 of 5 . The muscles in both distal lower limbs 
showed distinct atrophy. Deep tendon reflexes were normal except for a diminished Achilles tendon reflex. Babinski signs were absent. She could strive to go up and down the stairs by herself and walk without staggering. The CK was at 11145U/L. Magnetic resonance imaging (MRI) of the bilateral lower extremities revealed increased signal intensity in the gastrocnemius(fig1-A). Immunohistochemical staining of muscle specimens revealed a strongly reduced dysferlin expression (fig1-B). Sequencing of the DYSF gene revealed heterozygous mutations comprising chr2-71896273 exon49 c.5512A>T(p.R1838X)and a large-segment duplication at exon6-44(fig1-C,D). Family analysis showed that the c.5512A $>\mathrm{T}$ variant was of paternal origin and the exon6-44 large-segment duplication mutation was of maternal origin. 

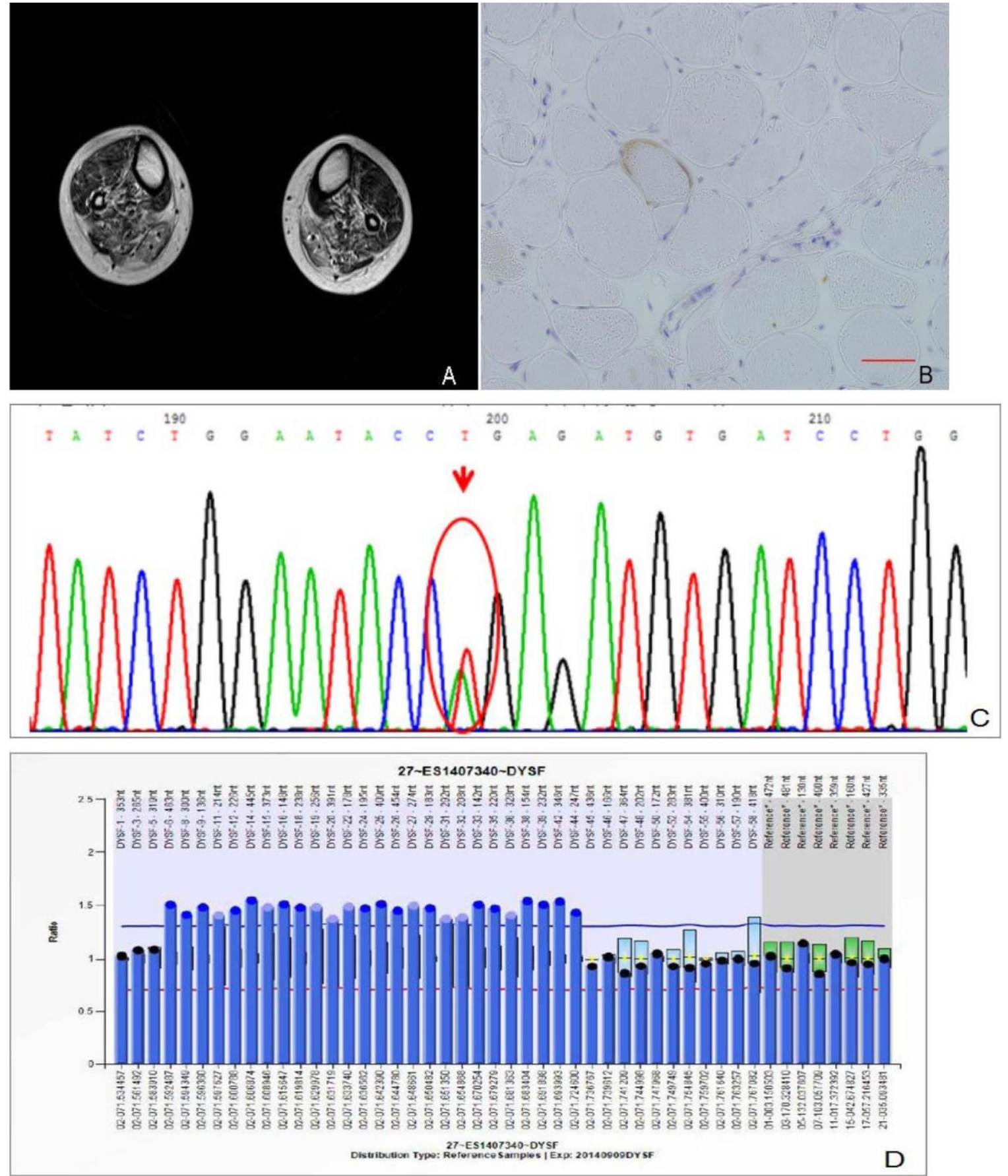

Fig. 1. MRI of the bilateral lower extremities revealed increased signal intensity in the gastrocnemius(A). Immunohistochemical staining of muscle biopsies revealed strongly reduced dysferlin expression(B). Sequencing of the DYSF gene revealed heterozygous mutations comprising chr2-71896273 exon49 c.5512A>T(p.R1838X)and a large-segment duplication in exon6-44 (C,D).

\section{Discussion}


We report a unique case of dysferlinopathy with heterozygous mutations comprising c.5512A $>$ T (p.R1838X) and a large-segment duplication in exon 6-44. It is worth noting that the patient's state was obviously aggravated after small-dosage steroid treatment. We propose the following possible explanations for this adverse reaction: 1 . The small amount of residual dysferlin protein in the muscles was reduced after methylprednisolone treatment, inducing muscle-membrane damage. A prospective, double-blind, placebo-controlled trial assessed the safety and efficacy of deflazacort treatment for dysferlinopathies[2]. Deterioration was observed in 6 patients during deflazacort treatment. Patients with residual dysferlin protein experienced more serious worsening than patients with total absence of dysferlin. It was hypothesized that steroids might have a negative effect on dysferlin expression or function. Steroids may have affected the patient's residual dysferlin protein expression so that her symptoms worsened and the CK levels rose dramatically. 2. Acute corticosteroid myopathy(ACM): Most cases of corticosteroid myopathy usually result from prolonged use of steroids and CK in these cases is normal. However, we noticed recent reports showing similar cases of acute myopathy after taking small amounts of glucocorticoids for a few days [3, 4]. Askari et al. concluded that the development of myopathy in patients receiving corticosteroid therapy is not related to the magnitude of the dose given or the duration of maintenance therapy[5]. Several theories have been proposed to explain the pathogenesis of ACM. One model proposes the activation of ubiquitin dependent proteolytic systems as the proximal pathophysiological driver[6]. Another model suggests that insulin-like growth factor-1, which may act as an anti-apoptotic signal, is inhibited by steroids, allowing increased muscle apoptosis[7]. Dysferlinopathy patients tend to display obvious spontaneous muscle apoptosis in biopsy samples. One case described a 15-year-old African-American boy with dysferlinopathy presenting as rhabdomyolysis[8]. Before steroid therapy, the patient was in the stable stage of dysferlinopathy. Steroid might be a stimulating factor and induce a negative effect on dysferlin expression or function. Therefore the persistent process may be the intrinsic progression of the dysferlinopathy after the corticosteroid usage.

To our best knowledge, this is the largest segment duplication mutation of DYSF which has been reported to date, and the first detailed case report of such a large-scale mutation. We 
have not found any literature about the relation of clinical features to DYSF duplication mutations. More work is needed on the dysfelin protein changes caused by the exon6-44 large-segment duplication mutation in order to explain the symptoms definitely.

The unusual reaction to small-dose steroid therapy highlights the importance of careful differential diagnosis of inflammatory myopathies and the necessity of ruling out dysferlinopathy before prescribing corticosteroids.

\section{Acknowledgments}

We are grateful to the subject for participation in our study. This work was supported by the grants from the Beijing Natural Science Foundation Program and Scientific Research Key Program of Beijing Municipal Commission of Education (No. KZ201410025023), the National Science Foundation of China (No. 81071000).

\section{References}

[1] Tarnopolsky MA, Hatcher E, Shupak R. Genetic Myopathies Initially Diagnosed and Treated as Inflammatory Myopathy. The Canadian journal of neurological sciences Le journal canadien des sciences neurologiques. 2016;43:381-4.

[2] Walter MC, Reilich P, Thiele S, Schessl J, Schreiber H, Reiners K, et al. Treatment of dysferlinopathy with deflazacort: a double-blind, placebo-controlled clinical trial. Orphanet journal of rare diseases. 2013;8:26.

[3] Kumar S. Steroid-induced myopathy following a single oral dose of prednisolone. Neurology India. 2003;51:554-6.

[4] Khan MA, Larson E. Acute myopathy secondary to oral steroid therapy in a 49-year-old man: a case report. Journal of medical case reports. 2011;5:82.

[5] Askari A, Vignos PJ, Jr., Moskowitz RW. Steroid myopathy in connective tissue disease. The American journal of medicine. 1976;61:485-92.

[6] Mitch WE, Goldberg AL. Mechanisms of muscle wasting. The role of the ubiquitin-proteasome pathway. The New England journal of medicine. 1996;335:1897-905.

[7] Singleton JR, Baker BL, Thorburn A. Dexamethasone inhibits insulin-like growth factor signaling and potentiates myoblast apoptosis. Endocrinology. 2000;141:2945-50.

[8] Moody S, Mancias P. Dysferlinopathy presenting as rhabdomyolysis and acute renal failure. Journal of child neurology. 2013;28:502-5. 